\title{
Contrasting Views of the Post-bariatric Surgery Experience between Patients and their Practitioners: a Qualitative Study
}

\author{
Sandra Jumbe ${ }^{1}$ (D) Jane Meyrick ${ }^{2}$
}

Published online: 12 March 2018

(C) The Author(s) 2018

\begin{abstract}
Background The superiority of obesity surgery for improving medical and weight outcomes in severely obese patients when compared to other weight loss interventions remains undisputed. However, knowledge about the psychological impact of the procedure on patients' lives is limited. Systematic reviews indicate persisting psychological distress after surgery compared to control groups especially longer term, suggesting the need for postoperative psychological support and assessment. Research literature also infers limited knowledge regarding the postoperative patient experience of obesity surgery. This may form a barrier in health practitioners' understanding of these patients' ongoing needs.

Methods Ten patients who had obesity surgery two or more years ago and eight obesity surgery practitioners were recruited within hospital settings and individually interviewed by the researcher to capture their accounts of the postoperative experience. Concordance between the two groups was explored to gauge awareness of patients' subsequent health needs.

Results Thematic analysis of transcribed interviews elicited a key finding around 'post-surgical cliffs in patient care' within a heavily structured service. Participants reported some unmet needs, namely, psychological aftercare to facilitate adjustment following drastic weight loss and excess skin, acceptance of their non-obese self and perceived stigma. The impact of contrasting views of success between patients and practitioners on postoperative care within the service context was highlighted.

Conclusions Obesity surgery is a great weight loss catalyst for severe obesity. However, lack of psychological aftercare may threaten early gains in health outcomes over the longer term. More qualitative and quantitative studies are needed to validate current study results.
\end{abstract}

Keywords Psychological health $\cdot$ Qualitative $\cdot$ Longer term outcomes

\section{Introduction}

The superiority of obesity surgery when compared to other weight loss interventions in relation to weight loss and improving medical outcomes in severely obese individuals remains undisputed [1-3]. However, current literature shows limited examination of psychological and social health

Sandra Jumbe

s.jumbe@qmul.ac.uk

1 Centre for Primary Care and Public Health, Barts \& The London School of Medicine and Dentistry, Queen Mary University of London, Yvonne Carter Building, 58 Turner Street, London E1 2AB, UK

2 Department of Psychology, University of the West of England, Frenchay Campus, Bristol BS16 1QY, UK outcomes following the procedure [4]. Obesity surgery is predominantly delivered and framed within a medical context despite evidence that obesity stems from combined biological, psychosocial and environmental factors $[5,6]$. This may be a reflection of the traditional approach of healthcare practitioners involved in the surgical intervention's process. Nevertheless, we may be overlooking patients' psychosocial needs that relate to the underlying causes of lifelong weight problems. Most quantitative research on the postoperative impact of obesity surgery focuses on physical outcomes such as weight loss and co-morbidities. Psychosocial outcome assessment is limited to pre-existing validated self-report outcome measures [4] with emphasis on screening for surgery, which restricts opportunity to capture detailed insights into patients' postoperative experience [7]. Some long-term studies show minimal postoperative improvements in psychological wellbeing compared to behavioural interventions and usual care despite significant improvements in physical quality of life, 
weight loss and co-morbidities $[4,8,9]$. This suggests a subset within the obesity surgery community that struggle psychologically despite generally positive medical and physiological outcomes [9]. These postoperative studies also show trends of weight regain typically after 2 years, implying that surgery alone may not be a sustainable obesity intervention [10]. Limited focus of biopsychosocial outcome assessment may be a barrier for practitioners understanding this patient group's postoperative needs. Research gaps suggest a need for richer data that encapsulates the lifelong disease trajectory of obesity within a psychological context and broadening of bariatric practitioners' perspectives to optimise patient health outcomes. [4].

Several qualitative studies have captured the postoperative patient experience of obesity surgery [11-13] and practitioners' experiences working with this patient group [14, 15]. Findings indicate that the physical restriction induced by the procedure provides an external control to weight and eating that patients previously lacked $[16,17]$, with reported new experiences of reduced hunger despite occasional negative feedback, e.g., nausea and pain $[18,19]$. However, it seems that this external control fades typically a year or two after surgery, and maintaining weight loss becomes a struggle, triggering lingering fears of weight regain [20]. Therefore, control over eating and other related health habits remains an ongoing psychological struggle as patients' postoperative journey progresses $[17,20]$. Studies also report emotional changes, such as more emotional distress when adjusting to physical changes and a 'non-obese' identity, following drastic weight loss over time [12, 21]. For example, some patients see their excess skin, flaccidity and scars from dramatic weight loss as therapeutic failures, resulting in a constant quest for plastic surgery and potentially reigniting body dissatisfaction [22].

These studies also illustrate the complex psychological processes faced by patients postoperatively when adapting to both positive and negative physiological changes, which warrants postoperative psychological support.

Qualitative studies specifically exploring practitioners' postoperative working experience with this patient group are sparse. One study found that registered nurses held positive attitudes toward obese adults, not only recognising the complex care needs of bariatric patients but also the increased workload associated with meeting these demands [15]. Findings from Whitfield and Grassley [14] who also interviewed nurses about their postoperative bariatric care experiences revealed challenges related to 'power struggles' between nurses, patients and families. Nurses described complexities of discerning between dependent and independent patients when providing support with ambulation. They also reported complex family dynamics whilst helping patients with self-care and eating, illustrating obstructive family behaviours like 'doing everything for their loved ones' which delayed recovery. Both studies suggest that postoperative bariatric care provision presents multifaceted challenges from medical, personal and social angles.

Reflecting on the current literature on psychological impact of obesity surgery, not much is known about whether patients think psychological support after obesity surgery is needed and, if so, what kind of support would be most amenable. This is despite qualitative research reporting notable changes that warrant psychological involvement $[11,23]$. We also know little about practitioners' experiences of providing bariatric care, and what they think is central to effective postoperative weight management [14]. As national clinical guidelines recommend psychological support as part of standard postoperative care, it would be particularly useful to explore patients and health practitioners' views around the nature of this psychological support. As such, this present study explored patients and their practitioners' accounts of the postoperative life experience of obesity surgery using a qualitative approach. Qualitative data from these two perspectives may suggest how to maximise the effectiveness of obesity surgery within a behavioural, health psychology informed perspective.

\section{Methods}

\section{Procedure}

This study received UK National Research Ethics and local R\&D approvals before starting recruitment in a regional National Health Service (NHS) Bariatric Service. Patients were recruited via convenience sampling. Those eligible were identified through the service's patient database $(N=94)$ and sent a postal invitation containing study information, a reply slip and prepaid envelope to return the slip.

Practitioners were recruited via snowballing, following a study talk by the researcher or email invitation from the NHS collaborator (which included a participant information sheet and a copy of the consent form attached).

Interested individuals either emailed, called or posted their reply slip to the researcher, who subsequently arranged an interview at a date and time convenient for potential participants. After obtaining written informed consent, the researcher conducted semi-structured interviews with participants supported by interview schedules (see Table 2). Interviews were audio taped and transcribed verbatim by the researcher within 3 days of being conducted. After each interview, participants completed a short demographics form. The researcher also debriefed each participant, reconfirming contact details and encouraging them to make contact should they have any subsequent concerns or queries concerning the research. 


\section{Participants}

Ten patient participants who had undergone obesity surgery at least 1 year ago and were over 18 years old were recruited (Table 1). Twelve patients initially responded to study invitations (two subsequently dropped out). Eight practitioners in a job that involved providing psychological or medical aftercare to obesity surgery patients were recruited. A ninth practitioner dropped out. Most participants (14 out of 18) opted for face to face interviews. Four participants opted for a telephone interview due to convenience (Table 2).

\section{Data Analysis}

Interview transcripts were analysed in accordance with Braun's and Clarke's thematic approach [24] which outlines essential six stages for a thematic analysis, providing a clear, well-defined explanation of what it is and how it is carried out whilst maintaining the 'flexibility' tied to its epistemological position (see Fig. 1). In line with outlined qualitative research recommendations, the acquired sample size is considered appropriate for a small to medium-sized thematic analysis study [24].

To increase rigour in the qualitative analysis process, ongoing feedback on themes was sought from the study team and NHS collaborator to verify analysis and general interpretation of data. The researcher also kept a reflective journal throughout the recruitment and data analysis process to increase transparency of preconceptions in the research and to aid critical self-reflection of the research process [25].

\section{Results}

The general characteristics of this patient cohort are reported in Table 1. The average age was 54 years, with most participants being 50 years old and above. There was only one male patient participant. These characteristics generally reflect the wider weight loss surgery patient group as reported by the recent UK National Bariatric Surgery Register where patients tend to be older and female [26]. Most of the group were in employment (56\%). Five participants stated they had a diploma or similar qualification; the others did not disclose information on educational background.

Recruited practitioners covered a range of roles, namely a physician, a surgeon, three psychologists, one bariatric practitioner and two dieticians, reflective of most vital clinical staff within an NHS bariatric surgery service. Besides formal clinical training, this group completed informal on the job shadowing to increase their professional clinical experience in obesity surgery. It was felt that 6 months as a minimum period working in this particular service was sufficient time for one to settle within a job role and start to gain experience of a particular patient group. Overall, with good representation of all roles present within the bariatric service multidisciplinary team (MDT) and length of bariatric working experience ranging from 18 months to 9 years, these eight health professionals had sufficient diverse perspectives and knowledge of this NHS service to allow them to give informative real life depictions of how the service operates and insight into patients' bariatric experience from their standpoint.

\section{Contrasting Perspectives Between Patients and Their Practitioners}

Two key underlying ideas emerged throughout the analysis and underpinned all the themes identified; a contrast between patient and health practitioners' perspectives of a shared service and a postoperative 'cliff' defined as a drop off in care (Fig. 2). The effect of these will be highlighted in the examination of three main themes, namely post-surgical expectations, measures of success and unmet patient needs, emerging from both patient and practitioner accounts. For illustration, quotes from participants' transcripts have been used alongside appropriate pseudonyms for the patient cohort. Codes instead of pseudonyms in the format $H P \#$ have been used for practitioners' quotes for increased anonymity.

\section{Post-Surgical Expectations}

An important finding reflected in patients and practitioners contrasting perspectives was the impact of miscommunication following surgery within the theme of post-surgical expectations. Both groups reported that support after obesity surgery was reliant on patients contacting the service for help. However, such support was perceived very differently by the two groups. In general, practitioners felt they maintained good channels of communication and all patients experiencing problems with their band or sleeve would contact the service as advised. Therefore, if they did not hear from a patient, they assumed the patient was fine. However, a difference in opinion regarding this approach was noted between practitioners. Those working more in acute surgical delivery felt the communication channels within the service were clear and good.

We kind of also have an open door policy where we are able to be contacted at any time during post op by patients if they are concerned or worried about anything. Our communication channels are very good. Patients can refer back into our service if there's things that they are worried about (HP4)

Practitioners who worked with patients over a longer period (predominantly psychologists and dieticians) felt that communication within the service was an ongoing issue, particularly after surgery. These practitioners shed light on the 
Table 1 Details of patient interviewees

\begin{tabular}{lllllll}
\hline Pseudonym & Age & Sex & Time since surgery & Type of Surgery & Weight loss since surgery & Discharged from service \\
\hline Sarah & 51 & F & 6 years & Gastric band & 8 stone & Yes \\
Pauline & - & F & 3 years 2 months & Gastric bypass & 7 stone & Yes \\
Hazel & 60 & F & 2 years & Gastric band & 4 stone & No \\
Gail & 53 & F & 2 years & Gastric sleeve & 7 stone & No \\
Frank & 56 & M & 2 years 3 months & Gastric band & 6 stone & Yes \\
June & 62 & F & 3 years & Gastric band & $91 / 2$ stone & Yes \\
Anna & 49 & F & 2 years 2 months & Gastric sleeve & 6 stone & No \\
Fran & 35 & F & 2 years 6 months & Gastric sleeve & $51 / 2$ stone & Yes \\
May & 60 & F & 2 years 2 months & Gastric band & Not disclosed & No \\
Harriet & 60 & F & 5 years & Gastric bypass & 8 stone & Yes \\
\hline
\end{tabular}

Information based on participant's transcript as demographic form not returned

complex NHS obesity surgery patient trajectory and the need for time and frequent consultation to assess patients' suitability for the procedure. They saw the preoperative phase as foundational to building good patient practitioner relationships and facilitating patients' motivation, empowerment and skills to make positive choices independently. In their view, solely weight-focused postoperative assessment was not enough to truly understand patient progress. Therefore, unlike their colleagues, they identified a need for long-term monitoring.

That gap between tier 3 and tier 4 I think communication between the two historically has been very poor (HP6) It helps to know them preoperatively and the whole way through to build a good relationship with them ... On the surface of things it looks like they've done amazingly but actually now she can't eat food properly she's turned to alcohol and is saving all her calories for alcohol. So you have to look below the surface of weight loss results to really know and hear how somebody is really doing (HP1)

Patient narratives mirrored the negative view of postoperative communication. A general lack of communication from the service was reported by some participants as leading to a reluctance to contact the service and seek help when they should have. In contrast to the intense input prior to surgery, this was experienced as a post-surgical cliff, with feelings of abandonment in patients from the point of surgery discharge.

Hmm there is the shortfall I would say in that the support you get afterwards is very little. Except for ... I had had no communication except for having fills. There was no real advice. I had one meeting with the dietician. My doctors followed up with some blood tests, that sort of thing to make sure that I was getting all the nutrients that I should. Uhm but for the follow up of advice and that sort of thing I had to find out myself which I found a little bit disappointing. (May, aged 60, 2 years since operation)

I kind of felt like you had the band and then they basically pushed you out of the door and said get on with it (Sarah, aged 51, 6 years since operation)

Afterwards I felt abandoned. A bit more aftercare would be better (June, aged 62, 3 years since operation)

Overall, despite some practitioners recognising the psychologically vulnerability of post-surgery patients, minimal or response only postoperative service models limited their ability to follow up patients. Practitioners therefore may be missing feedback about patients' ongoing physical and psychosocial health changes. Diversity of perspectives was noted not only between patients and their practitioners but also between practitioners with multidisciplinary teams.

\section{Measures of Success}

Contrasting perspectives were similarly identified throughout the theme around postoperative success measures. Accounts implied that practitioners felt that weight loss was the number one priority for patients, followed by assessment of comorbidities and medication intake, weight was reported as the main outcome measure of success.

For patients, that (weight) often is the most important thing, initially at least (HP5)

First and foremost weight... Physically, their other medical problems like diabetes and whether they are still required to take medications. Their functional ability where their mobility is concerned (HP4)

Narratives revealed a heavy focus on biomedical markers and a lack of postoperative psychological measures. This 
Table 2 Interview schedules

Patient interview schedule

1. Please tell me when you had weight loss surgery and the aftercare support you received after the procedure?

- Who was involved? How long was follow-up?

2. How has weight loss surgery affected your quality of life now that you are ... years post-op?

3. What is different for you now compared to the first year after weight loss surgery?

4. How has weight loss surgery impacted your (physical) health?

- Positives and negatives

5. How has weight loss surgery impacted the way you feel emotionally?

- Emotional / psychological health? Positives? Negatives?

6. Has weight loss surgery impacted your personal relationships?

- In what way?

- What (other) cultural/social implications you experienced since WLS?

7. Do you participate in any types of weight loss surgery support groups or online communities?

- If any...why OR if none... why not? What have you gained from them?

- Other sources of support?

8. Are there any other things you would like to say about weight loss surgery and how it has impacted your life that we have not covered?

9. Overall, are you happy with the results you have experienced from weight loss surgery?

10. Overall, how are you doing now?

- Would you say you feel in control of your weight? Eating? Physical activity?

Health professionals interview schedule

1. Please tell me about your role within the weight loss surgery team.

2. How is aftercare for a service user planned by yourself (and the Team) after they have had weight loss surgery?

3. On average how long would you be expected to see a service user for after they have had weight loss surgery?

4. Is the service user's aftercare reviewed within the team?

5. There is a term being used in health care now called Personalisation. For many people, personalisation is often seen as putting service users firmly in charge of their care and support and that care is designed with their full involvement and tailored to meet their own unique needs. Do you think your approach to aftercare and treatment planning with service users is personalised?

6. How do you consider issues of safety and risk for service users after they have had weight loss surgery?

7. Are family members/carers/friends involved in the aftercare process?

8 . How has the provision of weight loss surgery and aftercare changed over the years?

9. Do you think weight loss surgery is a good treatment for your service users in the long-term?

10. Can you suggest an intervention that would improve the aftercare provided to service users after having weight loss surgery?

11. Is there anything else you would like to say that we have not covered?

is in contrast to the proliferation of various preoperative psychological assessments used for screening risk and practitioners' recognition of an array of complex mental health problems within this patient group before and after obesity surgery. One practitioner's use of the word 'strategically' in the quote below when asking patients about their psychological problems may infer that they know that they do not have the resources for postoperative psychological support so they try not to ask.
We don't have any direct measures of emotional wellbeing post op. We don't strategically you know ask questions on that (HP6)

So when people talk about some of the pre op screening tools some of the questions are about (cause postoperatively people can suffer these symptoms) so we'll be asking questions about cutting or self-harming episodes because we find these behaviours increasing postoperatively. We see people developing alcohol problems postoperatively, obviously people using drugs, smoking going up. Sometimes people suddenly develop unhelpful or make unhelpful decisions about relationships (HP5)

Patients themselves reported weight loss as a key marker of successful surgery, perhaps partly due to experiencing dramatic changes in weight within the first year.

The first year was great. I lost about 4 stone and obviously that helped me a lot (Hazel, age 60, 2 years since operation)

However, their accounts also illustrated that being overweight was a small part of a bigger issue for most patients. Reasons for being overweight were rarely attributed to just overeating. Reduced mobility following physical injury and overeating for 'comfort' following psychological trauma or socially negative experiences were also reported. There was a strong sense from these patients that obesity surgery was their last attempt to gain control of their weight and hopefully fix their other life problems. Patient narratives revealed that addressing chronic obesity surgically also required a change in their mental attitude alongside physical or behavioural change. Therefore, success for them was not just about losing weight but improving functional and social ability too.

I was almost ashamed that I had to ask my 14 year old son to put socks on .... and the first time I did that on the bed, I just literally sat there and cried. You know, it's little things like that ... and it's just really good (Anna, age 49, 2 years 2 months since operation)

The weight loss is brilliant. Really good and I am much more active, much better, much more mobile and just having a great time taking on new activities, new hobbies and just much much much improved quality of life (Frank, age 56, 2 years 3 months since operation) I'm finding I'm going out more with my friends. I'm very lucky that I've always had a very close friendship group. We've know each other since we were very little and I go out with them more now (Fran, age 35, 21/2 years since operation)

This service's focus on biomedical measures to assess obesity surgery outcomes seemed reductionist leaving psychosocial 
Fig. 1 Six steps of a thematic analysis

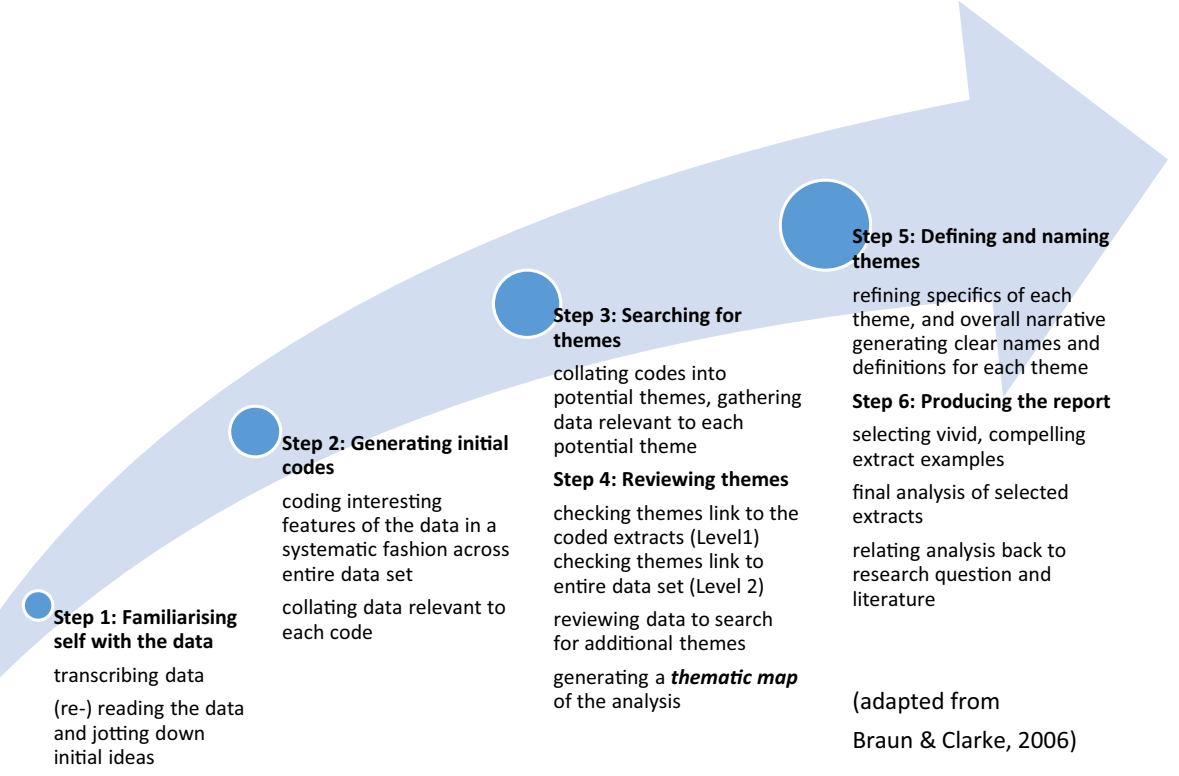

aspects of obesity unaddressed. This was experienced by patients as a post-surgical cliff where if weight loss was achieved, an assumption was made that the patient was doing well even if they may be struggling mentally. Conversely, patients who are struggling to lose or maintain weight, or have not experienced improvements in co-morbidities may feel like they have failed even if they have made gains elsewhere. Our knowledge of the psychological impact for this cohort remains neglected. Even data on other outcome measures tended to be short term, limited to the service's 2-year follow-up period.

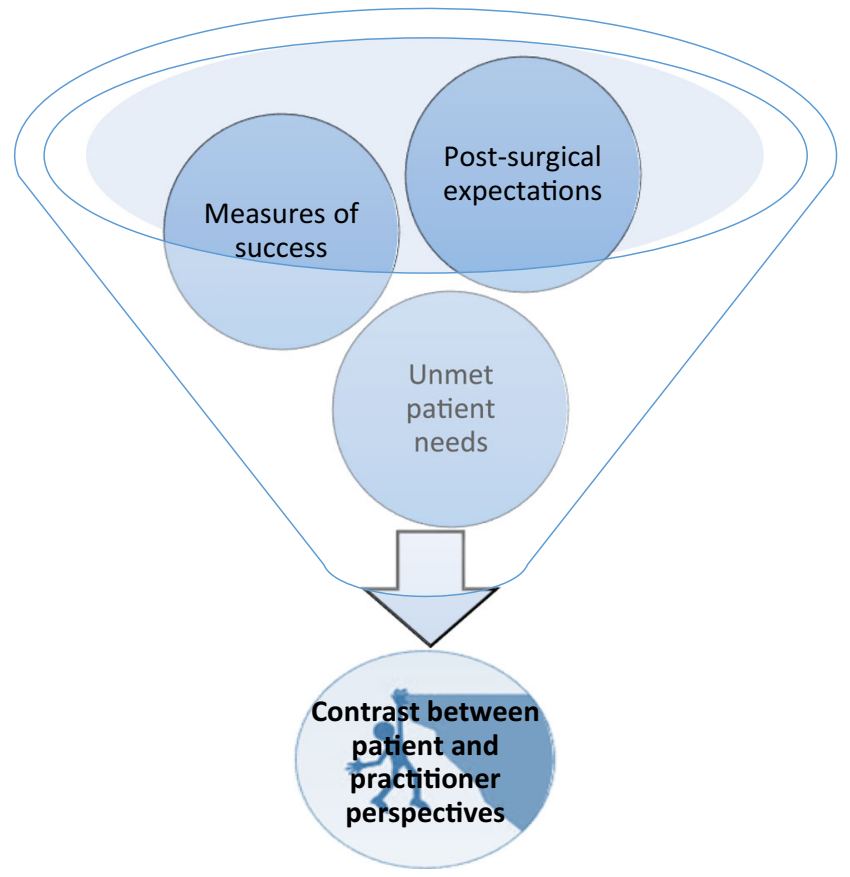

Fig. 2 Final Thematic Map
Overall, a need for widening the scope of progress markers beyond weight by including psychological and social factors was revealed which also roots recovery within the wider causes of obesity. There was also a sense that a joint patient-practitioner approach when assessing outcomes could facilitate concordance on success and the need to address ongoing progress beyond the current postsurgical cliff.

\section{Unmet Patient Needs}

Some patients felt that a lack of communication following discharge increased difficulties in dealing with unexpected physical and mental changes experienced after surgery. Examples of initial unexpected postoperative experiences included extreme pain whilst recovering from surgery and being sent home with injections without instructions on how to administer them.

Very early days after the operation, extremely painful. I never thought it would be that painful...I suppose when I think back I felt deserted after the operation because I was sent home with all these tablets, these injections and I was never sort of shown how to do the injections (June, aged 62, 3 years since operation)

One longer term issue reported by patients was excess skin following drastic weight loss. Overall patients felt that the service approach to patient discharge was so abrupt that it left little time for them to ask about aspects of the follow-up process that could not be anticipated prior to surgery. 
I know they're saying to me you've lost loads of weight but if you lose more what's the point? You've got all this loose skin and you're kind of feeling rubbish about that. It's going to affect things (Fran, age 35, 21/2 years since operation)

Unmet needs evident in patient interviews extended to postoperative psychological support being 'pushed out' by the acute weight-focused service approach. Only two patients saw psychologists after surgery within the service. Although most practitioners stated that postoperative psychology support was part of the official aftercare pathway, others clarified that this element was not standardised. Only patients identified by the multidisciplinary team as 'struggling' received psychological input postoperatively, but no formal assessment to identify them existed. The psychology team felt that this approach meant that they got to patients much later than desired.

It can be 6 months down the line that they'll be struggling and then we'd be asked by the team for us (psychologists) to see them but we don't follow up after surgery. The team are very good at picking people up and saying that they are struggling but normally we don't see people until something has gone wrong (HP2)

Psychologically, I think we could do a lot more. (HP6)

Practitioners empathised with patients' need for psychological aftercare, recognising the significant behavioural and psychological changes obesity surgery imposed on patients. Moreover, it was felt that this process of adjustment was ongoing and probably took longer than patient follow-up period in the service. Consequently, this care cliff caused by time limited aftercare may undermine longer term success of surgery. In fact, five practitioners reported witnessing an increase in revisional surgeries on patients 2 or 3 years following obesity surgery due to noncompliance with treatment plans and failure to manage dietetically after surgery.

Actually the reality is that they (patients) are only just settling after two years and the problem with the surgery is that old habits can creep in and the weight can regain, and if you've got no [psychological] support with that then the surgery might not be as successful as it could be. So I think, we keep talking about this. It's ongoing, support could be more (HP1)

This highlighted the need for more postoperative support. These unmet patient needs illustrate the interplay of competing priorities and perspectives, and the challenges practitioners encounter when trying to best address patient needs whilst following service protocol.

\section{Discussion}

Overall, this study's results suggest differing perspectives between patients and practitioners along the postoperative pathway resulting in unmet patient needs. A post-surgical cliff reflected a drop off in postoperative communication between staff and their patients, and limited provision of standardised postoperative psychological care. The perception of this cliff appeared to result from contrasting views between patients and practitioners regarding postoperative outcomes of success. A highly structured service also forced practitioners to focus on acute delivery of the procedure as an obesity intervention, leaving long-term issues unaddressed.

Participants' narratives revealed a lack of communication from the service soon after receiving the surgical intervention. Patients were not clear of the postoperative pathway despite their practitioners reporting a clear pathway existed. This lack of clarity reflects current ambiguity amongst NHS commissioning groups and relevant working bodies around the exact workings of the weight management tiered structure and how these tiers should feed into each other [27]. For instance, there is currently no universal geographical coverage of preoperative weight management services in the NHS [28] which sometimes results in overstretched teams that work across several specialist weight management teams as illustrated in practitioners' narratives. Ultimately, this structural complexity seems to cause patients distress, confusion, and at times feeling abandoned after their surgery whilst encountering physiological and psychological changes, thereby potentially jeopardising longer-term health gains. Greater clarity around service structure may improve practitioners working capacity and influence more strategic psychology aftercare across the board.

Findings also suggest service-led success measures with a narrow biomedical emphasis focused on weight loss and comorbidity outcomes. In this medical framing, patients attributed minimal or no weight loss as failure, exemplified by reported feelings of disappointment. Obesity surgery literature shows that although the procedure is the best evidence-based obesity treatment for weight loss and weight loss maintenance to date, $20-30 \%$ of people start to regain weight within 24 months [29]. Numbers of patients requiring revision surgery due to weight regain following their first procedures are growing with incident rates ranging from 5 to $56 \%$ [30, 31]. Gastric bands patients report higher revision surgery rates [32]. Perhaps, putting too much emphasis on weight as a health determinant and long-term progress marker in surgical obesity interventions may be misplaced [33]. Moreover, the reported absence of postoperative psychological measures in this service highlights the ongoing knowledge gap regarding postoperative long-term psychological disorders and trajectory in this patient group. This is despite the psychosocial challenges that the procedure has been shown to elicit [34]. 
Participants reported a lack of postoperative psychological support. The need for patients to directly ask or be assessed by the multidisciplinary team as struggling during follow-up consultations to access psychological aftercare poses an additional barrier. However, this may be a feature of this particular regional service. From a psychological context, literature shows that psychological illness mitigates against help seeking [35], more so in the context of obesity where stigma is rife $[36,37]$. As such, the ability to pick up struggling patients by waiting for them to ask for help is questionable. Minimal postoperative service-led communication may further isolate struggling patients, as they are less likely to seek help from the service.

Excess skin was reported to be problematic for most participants in this study, triggering feelings of shame and distress. Postoperative excess skin impacts approximately $70 \%$ of bariatric patients usually affecting the abdomen, arms, breasts and thighs, and can negatively affect ability to exercise [38]. Considering the appearance-related issues echoed by participants in this study when adjusting to changes, body image may be another postoperative outcome to prioritise within bariatric services [39]. Perhaps seeing bariatric outcomes in terms of body image may also improve practitioners' understanding of ongoing issues for this patient group [7].

The study findings, although limited to a small group, illustrate post-surgical cliffs, highlighted by unmet patient needs resulting from limited service provision. Practitioners' tendency to focus on delivery of the surgical intervention rather than the patients' life course perspective may amplify that cliff. Issues raised in this study point out the need to understand obesity surgery in the wider context of eating behaviour routes in early life, the function of eating as a coping mechanism and long-term behaviour change support needs of this patient group.

Lack of postoperative psychological assessment is another important issue because managing obesity should not be about attaining an ideal weight but addressing disordered eating behaviour. Evidence infers that weight loss interventions with psychological components like mindfulness or motivational interviewing reap better long-term and sustainable health benefits like increased activity, improved cardiovascular function and eating disorder even if individuals do not attain a healthy BMI [40-42]. Research also shows similarities in binge eating disorder psychopathology between obese and normal weight individuals implying that severity of the eating disorder is unrelated to weight $[43,44]$. Therefore, simply linking ideal weight to health, especially using BMI categorisation, is a reductionist perspective. Perhaps, 'degree of disordered eating' and 'psychological distress' are more appropriate outcome measures that could help capture psychosocial challenges along the obesity surgery journey, alongside weight and other physiological health outcomes.
Unfortunately, the narratives in this study seemingly raise challenges that may remain largely unsupported because current postoperative care, at least within this NHS service, seems minimal and based on patients' self-referral. According to the National Bariatric Surgery Register, about 45 NHS providers in England (including private providers) are currently commissioned to deliver obesity surgery. Capehorn and colleagues' recent obesity report highlights the issue of such inconsistencies in implementation of the NICE guidelines for obesity care and the resulting 'postcode lottery' effect regarding treatment provision across NHS England providers [45]. This implies that the experience of these participants may not be unique to this particular service.

To the authors' knowledge, this is the first qualitative study to explore patients and practitioners' post-bariatric experiences and perspectives. Other study strengths include the use of qualitative methodology to capture rich, detailed experiential information, alongside consistency of issues raised by patients in previous research $[20,22]$. Lastly, proportions of surgery type amongst the patient sample are representative of UK obesity surgery proportions reported in a recent epidemiology paper [46]. Study findings may be limited as participants are from one regional bariatric service. Therefore, more qualitative and larger scale quantitative studies are needed to validate the current study's findings and permit generalisabiity. Another limitation is that the patient sample only included one man potentially limiting the male perspective of the post-bariatric patient experience. Despite a 16-26\% increase in men seeking surgery in the UK from 2006 to 2013, the gender disparity where more women elect to have surgery still exists. This reduces the natural pool of men from the bariatric community who are eligible and willing to participate, as reflected in similar research studies [21, 22].

Overall, both practitioner and patient accounts highlight obesity surgery as a great catalyst that facilitates weight loss in severe obesity but has limited ability to sufficiently address underlying behavioural conditions that cause overeating and result in weight gain. This study also suggests a need, within one service at least, for more established postoperative psychology support irrespective of weight loss to address key physical and psychological issues, like emotional readjustment and impact of excess skin. Greater integration of health psychology into obesity surgery could facilitate a much needed shift toward biopsychosocial framing of progress markers and development of effective interventions that incorporate a life course perspective, alongside expertise of other health practitioners working in this area.

Acknowledgments The authors thank individuals, other than authors, who directly participated in the work, Professor Diana Harcourt, Centre for Appearance Research, University of the West of England, Frenchay Campus, Bristol, BS16 1QY, Dr. Emma Lishman, Clinical Psychologist, North Bristol NHS Trust, who was the study local collaborator. 


\section{Compliance with Ethical Standards}

Ethical Approval All procedures performed in studies involving human participants were in accordance with the ethical standards of the institutional and/or national research committee and with the 1964 Helsinki declaration and its later amendments or comparable ethical standards.

Informed Consent Informed consent was obtained from all individual participants included in the study.

Conflict of Interest The authors declare that they have no conflict of interest.

Disclosure Dr. Jumbe and Dr. Meyrick have nothing to disclose.

Open Access This article is distributed under the terms of the Creative Commons Attribution 4.0 International License (http:// creativecommons.org/licenses/by/4.0/), which permits unrestricted use, distribution, and reproduction in any medium, provided you give appropriate credit to the original author(s) and the source, provide a link to the Creative Commons license, and indicate if changes were made.

\section{References}

1. Herpertz S, Kielmann R, Wolf A, et al. Do psychosocial variables predict weight loss or mental health after obesity surgery? A systematic review. Obesity. 2004;12(10):1554-69.

2. Colquitt JL, Picot J, Loveman E, Clegg AJ. Surgery for obesity. Cochrane Database Syst Rev 2009; 2(2).

3. Colquitt JL, Pickett K, Loveman E, Frampton GK. Surgery for weight loss in adults. The Cochrane Library 2014.

4. Jumbe S, Bartlett C, Jumbe SL, et al. The effectiveness of bariatric surgery on long term psychosocial quality of life - a systematic review. Obes Res Clin Pract. 2016;10(3):225-42.

5. Vandenbroeck I, Goossens J, Clemens M. Foresight. Tackling obesities: future choices-building the Obesity System Map Government Office for Science, UK Government's Foresight Programme 2009.

6. Marks DF. Homeostatic theory of obesity. Health Psychol Open. 2015;2(1):2055102915590692.

7. Jumbe S, Hamlet C, Meyrick J. Psychological aspects of bariatric surgery as a treatment for obesity. Curr Obes Rep. 2017;6(1):71-8.

8. Karlsson J, Taft C, Rydén A, et al. Ten-year trends in health-related quality of life after surgical and conventional treatment for severe obesity: the SOS intervention study. Int J Obes. 2007;31(8):124861.

9. Herpertz S, Müller A, Burgmer R, et al. Health-related quality of life and psychological functioning 9 years after restrictive surgical treatment for obesity. Surg Obes Relat Dis. 2015;11(6):1361-70.

10. Adams TD, Davidson LE, Litwin SE, et al. Health benefits of gastric bypass surgery after 6 years. JAMA. 2012;308(11):1122-31.

11. Meana M, Ricciardi L. Obesity surgery: stories of altered lives. University of Nevada Press; 2008.

12. Warholm C, Øien AM, Råheim M. The ambivalence of losing weight after bariatric surgery. International Journal of Qualitative Studies on Health and Well-being 2014 01/29; 9.

13. Magdaleno R, Chaim EA, Turato ER. Understanding the life experiences of Brazilian women after bariatric surgery: a qualitative study. Obesity Surg. 2010;20(8):1086-9.

14. Whitfield PJ, Grassley J. Nurses' experiences of caring for postoperative bariatric patients. Bariatric Nursing Surg Patient Care. 2008;3(4):291-8.
15. Zuzelo PR, Seminara P. Influence of registered nurses' attitudes toward bariatric patients on educational programming effectiveness. The Journal of Continuing Education in Nursing. 2006;37(2):65-73.

16. Ogden J, Avenell S, Ellis G. Negotiating control: patients' experiences of unsuccessful weight-loss surgery. Psychol Health. 2011;26(7):949-64.

17. Engström M, Forsberg A. Wishing for deburdening through a sustainable control after bariatric surgery. Int J Qual Stud Health WellBeing. 2011;6(1):5901.

18. Ogden J, Clementi C, Aylwin S, et al. Exploring the impact of obesity surgery on patients' health status: a quantitative and qualitative study. Obesity Surg. 2005;15(2):266-72.

19. Ogden J, Clementi C, Aylwin S. The impact of obesity surgery and the paradox of control: a qualitative study. Psychol Health. 2006;21(2):273-93.

20. Natvik E, Gjengedal E, Råheim M. Totally changed, yet still the same: patients' lived experiences 5 years beyond bariatric surgery. Qual Health Res. 2013;23(9):1202-14.

21. Faccio E, Nardin A, Cipolletta S. Becoming ex-obese: narrations about identity changes before and after the experience of the bariatric surgery. J Clin Nurs. 2016;25(11-12):1713-20.

22. Magdaleno R, Chaim EA, Pareja JC, et al. The psychology of bariatric patient: what replaces obesity? A qualitative research with Brazilian women. Obesity Surg. 2011;21(3):336-9.

23. NICE Guidelines. Obesity: identification, assessment and management of overweight and obesity in children, young people and adults (CG189) 2014

24. Braun V, Clarke V. Using thematic analysis in psychology. Qual Res Psychol. 2006;3(2):77-101.

25. Ortlipp M. Keeping and using reflective journals in the qualitative research process. Qual Rep. 2008;13(4):695-705.

26. Welbourn R, Small P, Finlay I, Sareela A, Somers S, Mahawar K. The United Kingdom National Bariatric Surgery Registry. Second Registry Report 2014. 2014.

27. Welbourn R, Dixon J, Barth JH, et al. NICE-accredited commissioning guidance for weight assessment and management clinics: a model for a specialist multidisciplinary team approach for people with severe obesity. Obesity Surg. 2016;26(3):649-59.

28. British Obesity and Metabolic Surgery Society. Commissioning guide: Weight assessment and management clinics (tier 3). BOMSS 2014

29. Weineland S, Arvidsson D, Kakoulidis TP, et al. Acceptance and commitment therapy for bariatric surgery patients, a pilot RCT. Obes Res Clin Pract. 2012;6(1):e21-30.

30. Kellogg TA. Revisional bariatric surgery. Surg Clin North Am. 2011;91(6):1353-71.

31. Shimizu H, Annaberdyev S, Motamarry I, et al. Revisional bariatric surgery for unsuccessful weight loss and complications. Obesity Surg. 2013;23(11):1766-73.

32. Courcoulas AP, Christian NJ, Belle SH, et al. Weight change and health outcomes at 3 years after bariatric surgery among individuals with severe obesity. JAMA. 2013;310(22):2416-25.

33. Barth JH, O'kane M. Obesity services: how best to develop a coherent way forward. Clin Endocrinol. 2016;84(3):321-4.

34. Bagdade PS, Grothe KB. Psychosocial evaluation, preparation, and follow-up for bariatric surgery patients. Diabetes Spectrum. 2012;25(4):211-6.

35. Henderson C, Evans-Lacko S, Thornicroft G. Mental illness stigma, help seeking, and public health programs. Am J Public Health. 2013;103(5):777-80.

36. Poon M, Tarrant M. Obesity: attitudes of undergraduate student nurses and registered nurses. J Clin Nurs. 2009;18(16):2355-65.

37. Flint SW, Hudson J, Lavallee D. UK adults' implicit and explicit attitudes towards obesity: a cross-sectional study. BMC Obesity. 2015;2(1):1 
38. Baillot A, Asselin M, Comeau E, et al. Impact of excess skin from massive weight loss on the practice of physical activity in women. Obesity Surg. 2013;23(11):1826-34.

39. Coulman KD, Howes N, Hopkins J, et al. A comparison of health professionals' and patients' views of the importance of outcomes of bariatric surgery. Obes Surg. 2016;26(11):2738-46.

40. Daubenmier J, Moran PJ, Kristeller J, et al. Effects of a mindfulness-based weight loss intervention in adults with obesity: a randomized clinical trial. Obesity. 2016;24(4):794-804.

41. Donini LM, Cuzzolaro M, Gnessi L, et al. Obesity treatment: results after 4 years of a Nutritional and Psycho-Physical Rehabilitation Program in an outpatient setting. Eating and Weight DisordersStudies on Anorexia, Bulimia and Obesity. 2014;19(2):249-60.

42. Hardcastle SJ, Taylor AH, Bailey MP, et al. Effectiveness of a motivational interviewing intervention on weight loss, physical activity and cardiovascular disease risk factors: a randomised controlled trial with a 12-month post-intervention follow-up. Int J Behav Nutr Phys Act. 2013;10(1):40.

43. Dingemans AE, van Furth EF. Binge eating disorder psychopathology in normal weight and obese individuals. Int J Eat Disord. 2012;45(1):135-8.

44. Goldschmidt AB, Grange D, Powers P, et al. Eating disorder symptomatology in normal-weight vs. obese individuals with binge eating disorder. Obesity. 2011;19(7):1515-8.

45. Capehorn MS, Haslam DW, Welbourn R. Obesity treatment in the UK health system. Curr Obes Rep. 2016;5(3):320-6.

46. Booth HP, Khan O, Fildes A, et al. Changing epidemiology of bariatric surgery in the UK: cohort study using primary care electronic health records. Obes Surg. 2016;26(8):1900-5. 